\title{
Couleurs et dorures du portail roman de Cluny III. Restitution en 3D d'une œuvre disparue
}

Juliette Rollier-Hanselmann et Stéphanie Castandet

\section{(2) OpenEdition}

Édition électronique

URL : https://journals.openedition.org/cem/11601

DOI : 10.4000/cem. 11601

ISSN : 1954-3093

Éditeur

Centre d'études médiévales Saint-Germain d'Auxerre

Édition imprimée

Pagination : 235-250

ISSN : 1623-5770

Référence électronique

Juliette Rollier-Hanselmann et Stéphanie Castandet, «Couleurs et dorures du portail roman de Cluny III. Restitution en 3D d'une œuvre disparue », Bulletin du centre d'études médiévales d'Auxerre | BUCEMA [En ligne], 14 | 2010, mis en ligne le 18 octobre 2010, consulté le 22 septembre 2022. URL http://journals.openedition.org/cem/11601 ; DOI : https://doi.org/10.4000/cem.11601

Ce document a été généré automatiquement le 22 septembre 2022.

\section{c) (i) (8)(2)}

Creative Commons - Attribution - Pas d'Utilisation Commerciale - Partage dans les Mêmes Conditions 4.0 International - CC BY-NC-SA 4.0

https://creativecommons.org/licenses/by-nc-sa/4.0/ 


\title{
Couleurs et dorures du portail roman de Cluny III. Restitution en 3D d'une œuvre disparue
}

\author{
Juliette Rollier-Hanselmann et Stéphanie Castandet
}

1 La construction de l'ancienne abbaye Saint-Pierre-et-Saint-Paul de Cluny s'est échelonnée dans le temps, avec une première consécration du chevet en 1095, et une autre dédicace en 1130, correspondant vraisemblablement à une nouvelle étape d'avancement de la basilique romane.

2 Le portail, qui s'ouvrait sur la nef centrale, daté entre 1100 et 1120 , figure parmi les créations romanes de grande envergure qui nécessita des techniques exceptionnelles. Il mesurait 5,60 m de large et 3,25 $\mathrm{m}$ de hauteur, pour une épaisseur de $40 \mathrm{~cm}^{1}$. Selon la description de Benoît Dumolin au XviII ${ }^{\mathrm{e}}$ siècle $^{2}$, le tympan aurait été sculpté dans un bloc monolithe, tout comme le linteau, ce qui constitue une véritable prouesse technique, d'autant plus qu'il n'y avait pas de trumeau.

3 La poudre explosive, utilisée pour accélérer la destruction de l'abbaye au début du XIX ${ }^{e}$ siècle, a fortement dégradé le portail roman; les morceaux de la façade ont été retrouvés dans les remblais de la rue qui traversait l'avant-nef; seuls 5 à $10 \%$ du portail nous sont parvenus. Certains fragments ont été mis au jour par K. J. Conant dans les années 1930, et plus de 6000 fragments provenant de toutes les parties de l'avant-nef ont été sauvés de l'oubli en 1989 lors du déblaiement de ce secteur ${ }^{3}$.

\section{Iconographie}

Quelques éléments iconographiques permettent d'attester la présence d'un Christ en Majesté sur le tympan de Cluny III, sans pouvoir donner beaucoup de précisions ${ }^{4}$.

5 La gravure de P.F. Giffart (entre 1685-1713) montre le Christ, dans une mandorle, entouré par des anges ${ }^{5}$. Le dessin de J.-B. Lallemand, réalisé vers 1773, permet de confirmer la présence d'un Christ en Majesté, entouré de personnages, sans que l'on 
puisse préciser leur identité ${ }^{6}$. Le linteau, à peine esquissé, prend presque la forme d'un entablement. Pour l'archivolte, nous distinguons des arcatures contenant des éléments en relief difficiles à identifier. La restitution des piédroits est très aléatoire et ne nous aide guère. Le dessin d'A. Dauzat ( $\mathrm{XIX}^{\mathrm{e}}$ siècle) est une copie maladroite d'après Lallemand ${ }^{7}$.

6 L'archéologue américain K. J. Conant pensait que le centre du linteau était occupé par une Vierge entourée de deux anges, avec de part et d'autre deux groupes de six apôtres. Pour les extrémités du linteau, il propose la visite des saintes femmes au tombeau, à gauche, et les pèlerins d'Emmaüs, à droite, hypothèse qui reste difficile à vérifier en ce qui concerne les deux scènes latérales.

7 La typologie générale du portail rappelle d'autres œuvres proches de Cluny, notamment Anzy-le-Duc, Saint-Paul-de-Varax, ou encore Perrecy-les-Forges. Le portail roman de Cluny figure parmi les premières expériences de sculpture monumentale de grande envergure dans la région, ce qui a dû poser un certain nombre de contraintes techniques nouvelles. La datation se situe entre 1100-1120 selon les chercheurs.

\section{L'étude des polychromies}

8 La seule étude archéologique qui existe actuellement du portail roman est celle de K. J. Conant et H. Kleinschmidt, dont la restitution aquarellée (fig. 1) marquait, en quelque sorte, l'aboutissement de leurs recherches ${ }^{8}$.

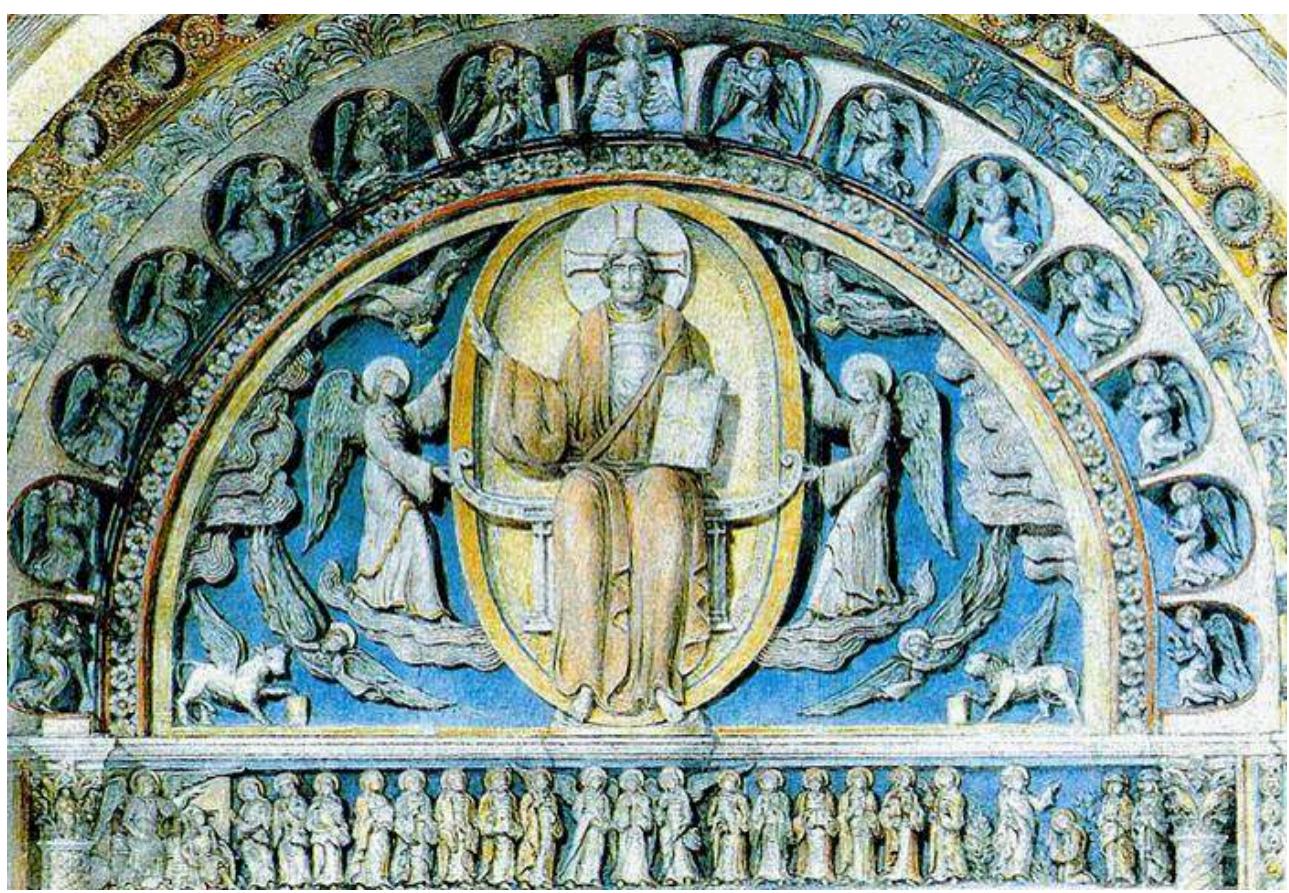

Fig. 1 - Le grand portail de Cluny III, reconstitution d'Helen Kleinschmidt (photo J.-C. Couval).

9 Cette aquarelle montre un portail qui a déjà perdu une grande partie de ses polychromies, de nombreux éléments étant à pierre nue. Les chercheurs ont constaté l'utilisation d'une palette riche aux couleurs vives - rouges, jaunes, bleu, blanc, noir, gris, brun roux, rose et dorure -, sans distinguer clairement les couches et les matériaux employés. Certains fragments conservaient davantage de couleurs, comme le grand morceau provenant de la mandorle du Christ ${ }^{9}$, à la base du tympan. Ce fragment, 
entreposé dans l'entrée d'une réserve du musée, a malheureusement fortement souffert des variations climatiques, provoquant la perte de la majeure partie des couleurs. Ce constat peut s'appliquer à la plupart des autres fragments. L'étude américaine constitue de ce fait un outil précieux pour évaluer la dégradation des couleurs durant les dernières décennies.

10 Les observations d'Helen Kleinschmidt étaient basées sur l'analyse de quelque soixante fragments ${ }^{10}$. Les diverses campagnes de fouilles effectuées depuis 1988 ont enrichi sensiblement le fonds lapidaire de l'avant-nef, ce qui nous permet maintenant d'étudier le portail roman sur la base d'environ 400 fragments (fig. 2). Seule une toute petite partie de ce fonds est présentée au public (environ 20 fragments).

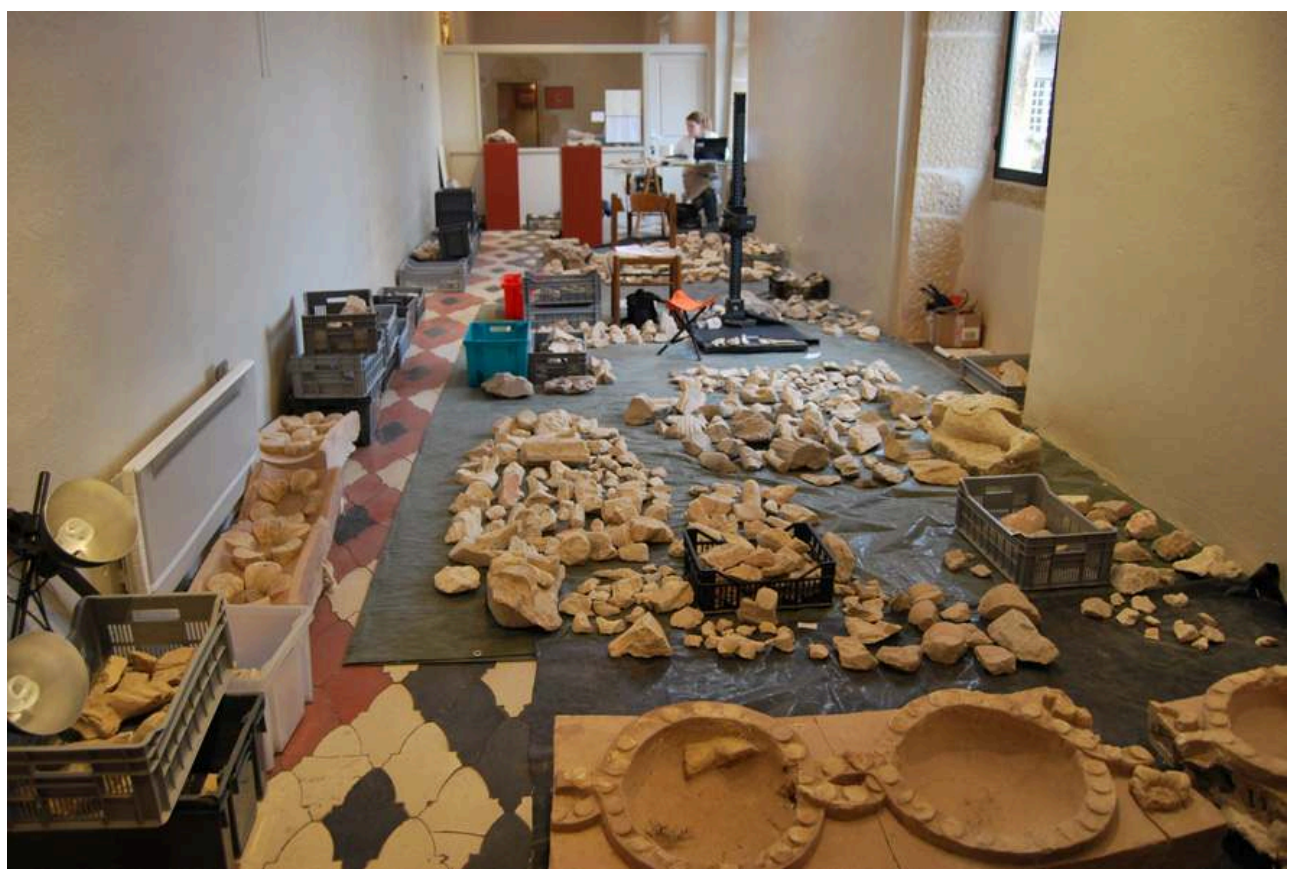

Fig. 2 - Salle de travail au Musée Ochier, vue générale des fragments (en avant-plan des fragments inclus dans du plâtre par K. J. Conant).

11 Entre 2009 et 2010, un financement exceptionnel nous a permis de trier le matériel découvert dans l'avant-nef et de classer le tout en proposant une analyse détaillée des stratigraphies picturales. Ce projet s'inscrit dans le cadre une restitution virtuelle en 3D de l'abbaye, qui permettra de montrer comment l'entrée de la plus grande abbaye de la chrétienté était marquée par une composition monumentale colorée ${ }^{11}$.

Pour le film diffusé au grand public, il a fallu trouver un compromis entre l'hypothèse de Conant et les fragments réellement conservés. L'ancienne maquette en plâtre a été scannée en 3D et retravaillée, de façon à fournir une image en relief du portail. La restitution colorée se base sur les résultats d'analyses physico-chimiques réalisées à l'IRAMAT-CRP2A ${ }^{12}$; l'étude n'étant pas achevée, l'article ne publie qu'un bref aperçu des résultats. 


\section{Le tympan (fig. 3)}

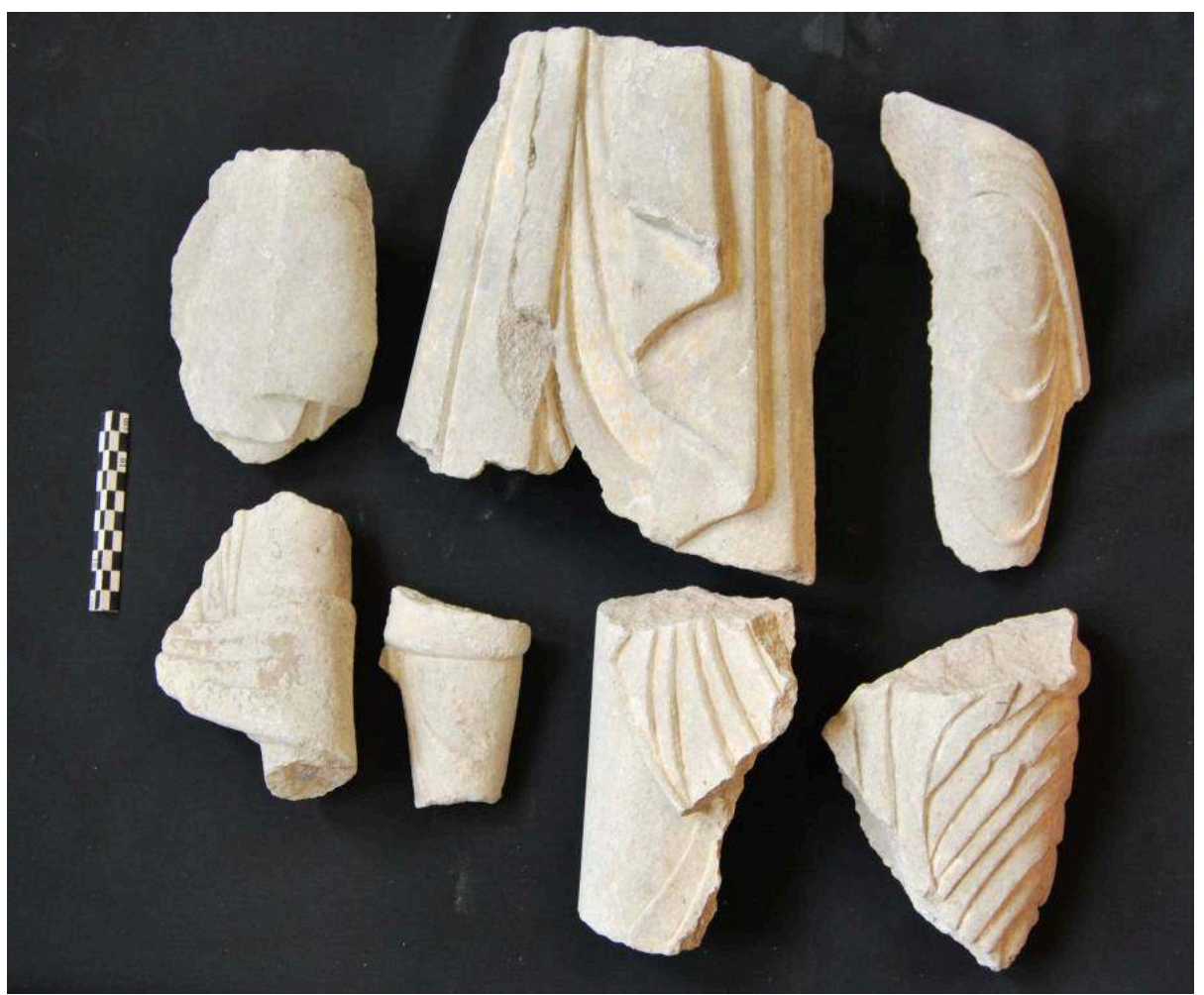

Fig. 3 - Cluny, portail roman, fragments du tympan (cl. J. Rollier).

Selon la description d'Helen Kleinschmidt, le Christ aux cheveux ocre, presque roux, portait un manteau aux plis épais de couleur ocre et rehaussés de vert, dont les bordures étaient ornées de motifs dorés. Malheureusement, il manquait alors une étude stratigraphique, ce qui nous prive d'un certain nombre d'informations. Il est toutefois possible de supposer un manteau peint en imitation de la pourpre, avec un glacis vermillon posé sur une sous-couche rouge. La description indique que la tunique du Christ était de teinte ocre sur une préparation blanche, ce qui permet d'imaginer une tonalité plus claire, en contraste avec le manteau sombre.

Le fond de la mandorle pose également le problème de la stratigraphie, car Kleinschmidt observait une couche jaune sur rouge sur préparation blanche. Doit-on y voir une couche romane et un repeint?

Nous pouvons y ajouter quelques éléments nouveaux, notamment un morceau appartenant peut-être à l'encolure du Christ, qui conserve des restes roses sur une préparation jaune ${ }^{13}$. Pour les nuées qui entourent la mandorle, certains fragments ${ }^{14}$ présentent des zones colorées gris-noir que l'on peut prendre aisément pour des salissures, voire des altérations, mais qui sont des restes de feuilles d'étain.

16 L'utilisation de ce matériau permet d'émettre plusieurs hypothèses. L'étain pouvait être utilisé en imitation de la feuille d'or ou de la feuille d'argent, par souci d'économie. La feuille d'étain pouvait également être vernie, de façon à ressembler à l'or, comme le préconise le moine Théophile au XII ${ }^{e}$ siècle ${ }^{15}$. Dans certains cas, l'étain est recouvert d'une feuille d'or à l'aide d'un mordant ${ }^{16}$ ou en battant les feuilles ensemble, technique déjà observée à Angers et Poitiers (XII siècle) ${ }^{17}$. L'utilisation de différentes techniques 
dorées était fréquente pour diversifier les éclats métalliques et obtenir des nuances à l'intérieur de l'œuvre.

17 Pour le tympan de Cluny, l'état de conservation ne permet plus de dire s'il y avait une différence entre les dorures du Christ et celles des anges. Quant aux voussures qui surplombent l'ensemble, nous observons une stratigraphie différente, ce qui est peutêtre dû au calcaire plus blanc et plus tendre. Ici les nimbes des anges sont dorés à la feuille d'or sur une préparation rouge.

D'une façon générale, Helen Kleinschmidt considérait que tous les fonds derrière les éléments figurés du tympan (anges, symboles des évangélistes) étaient colorés en bleu, ce que nous pouvons vérifier à partir de plusieurs fragments. Les analyses ont permis de déterminer la présence de lapis-lazuli.

Les quatre anges autour de la mandorle étaient vêtus de drapés ocre, blanc et rose selon Kleinschmidt, éléments qui sont devenus extrêmement ténus depuis. Des restes d'ailes de ces mêmes anges indiquent une coloration verte. Quelques fragments de visages, de mains et de pieds attestent d'une coloration jaune.

L'étude des symboles des évangélistes pose plusieurs difficultés. Si l'aigle de saint Jean est bien conservé (musée du Louvre) ${ }^{18}$, il ne présente plus aucune trace colorée, ce qui est dû à son dépôt dans un jardin ${ }^{19}$. Les autres symboles des évangélistes ne subsistent que sous forme de fragments. À l'époque de Kleinschmidt, le lion et le taureau conservaient encore des traces jaunes, qui sont devenues très ténues et pulvérulentes. Un morceau a été attribué à la tête du lion de saint Marc et atteste des restes rouges sur un apprêt blanc ${ }^{20}$.

21 Une patte tenant un livre appartient à un symbole d'évangéliste, peut-être le lion ${ }^{21}$, tandis qu'une autre patte d'animal pourrait provenir du taureau de saint Luc. Ce morceau conserve deux couches peintes, l'une probablement romane, l'autre postérieure. La coloration semble avoir été très simple, d'une teinte jaune uniforme, avec quelques tracés rouges autour des membres de l'animal, de façon à faire ressortir les volumes contre un fond bleu vif. Les sculptures ont été reprises par la suite, comme le montrent les restes d'un repeint jaune vif, également présent sur le lion. Il s'agit de stannate de plomb, de couleur semblable à l'orpiment, dont l'utilisation n'est pas attestée avant $1300^{22}$, pigment que l'on retrouve également sur des fragments appartenant à la façade gothique. 


\section{Le linteau (fig. 4 à 7)}

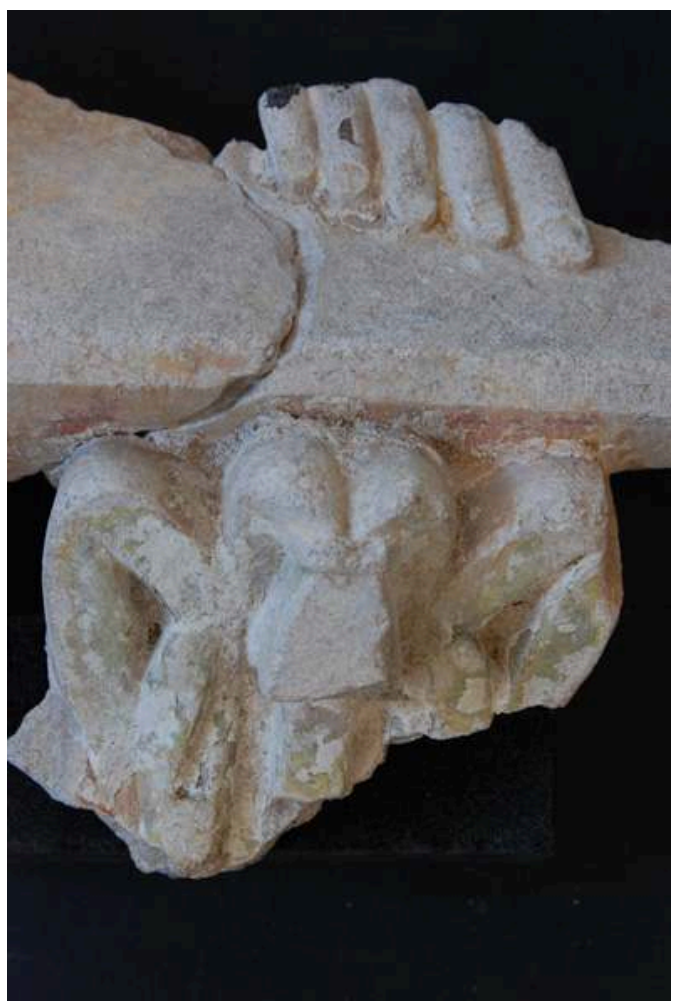

Fig. 4 - Fragment du linteau : pied d'apôtre et frise d'acanthe. La stratigraphie complète est visible sur les feuillages (cl. S. Castandet).

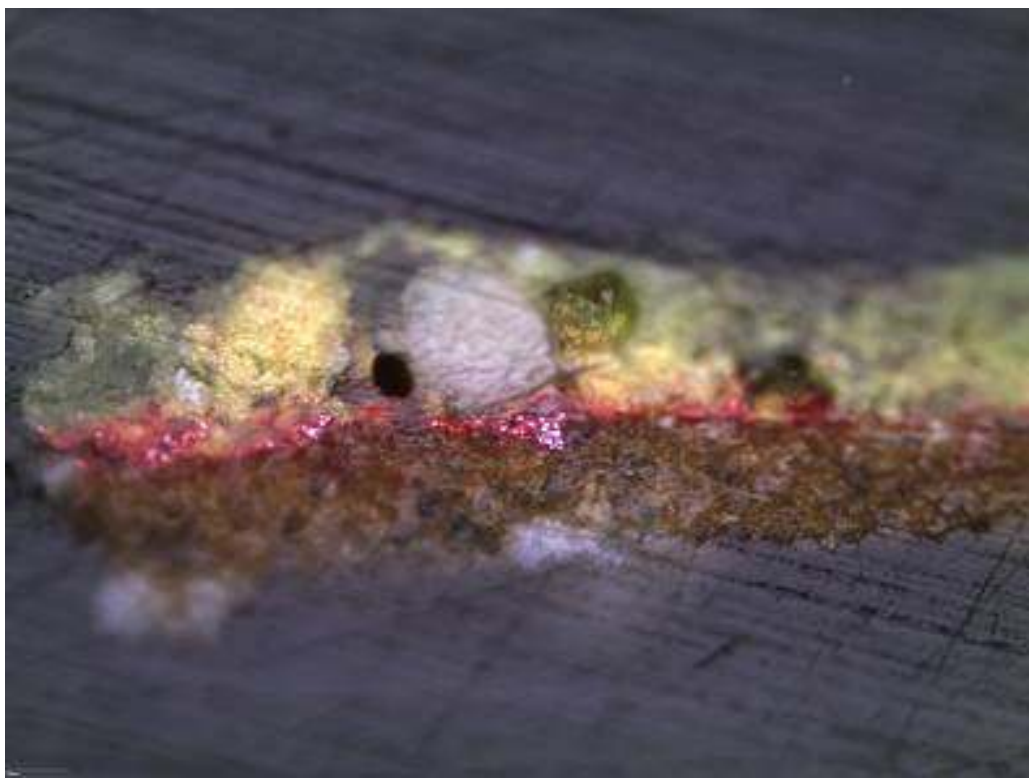

Fig. 5 - Coupes stratigraphiques provenant de prélèvements effectués sur le fragment de linteau bordé d'une frise d'acanthe (cl. S. Castandet).

En haut, échantillon provenant de la feuille d'acanthe : une couche verte de pigment au cuivre avec des rehauts jaune de stannate de plomb sur une couche de cinabre sur une couche préparatoire jaune.

En bas, échantillon provenant du fond des acanthes : couche de cinabre (gros cristaux rouge profond) posé sur un mélange de minium et de blanc de plomb (organge) posé sur une couche d'ocre jaune. 


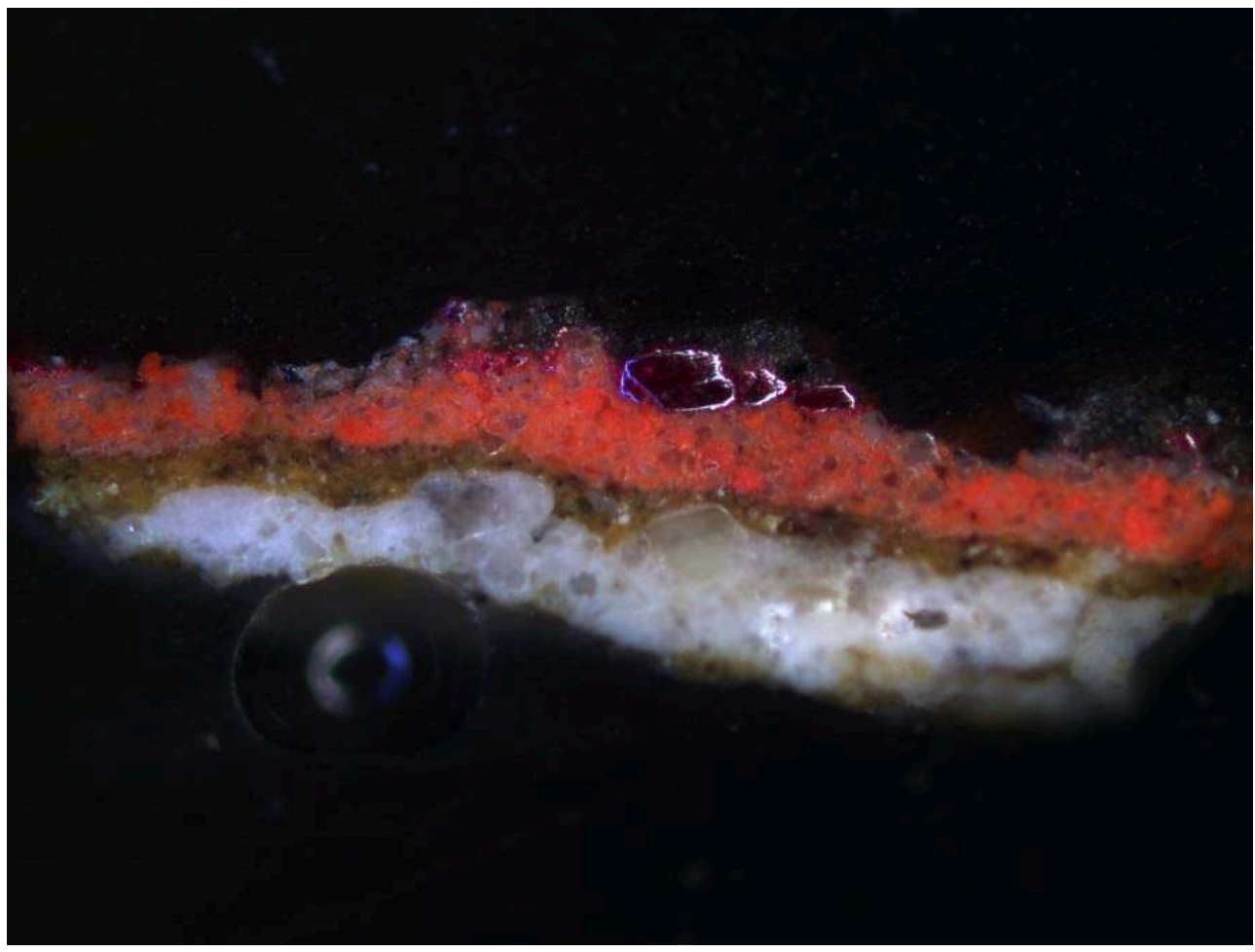

22 Pour le linteau, nous disposons d'une soixantainede fragments qui s'ajoutent aux neuf apôtres présentés dans les vitrines du musée ${ }^{23}$.

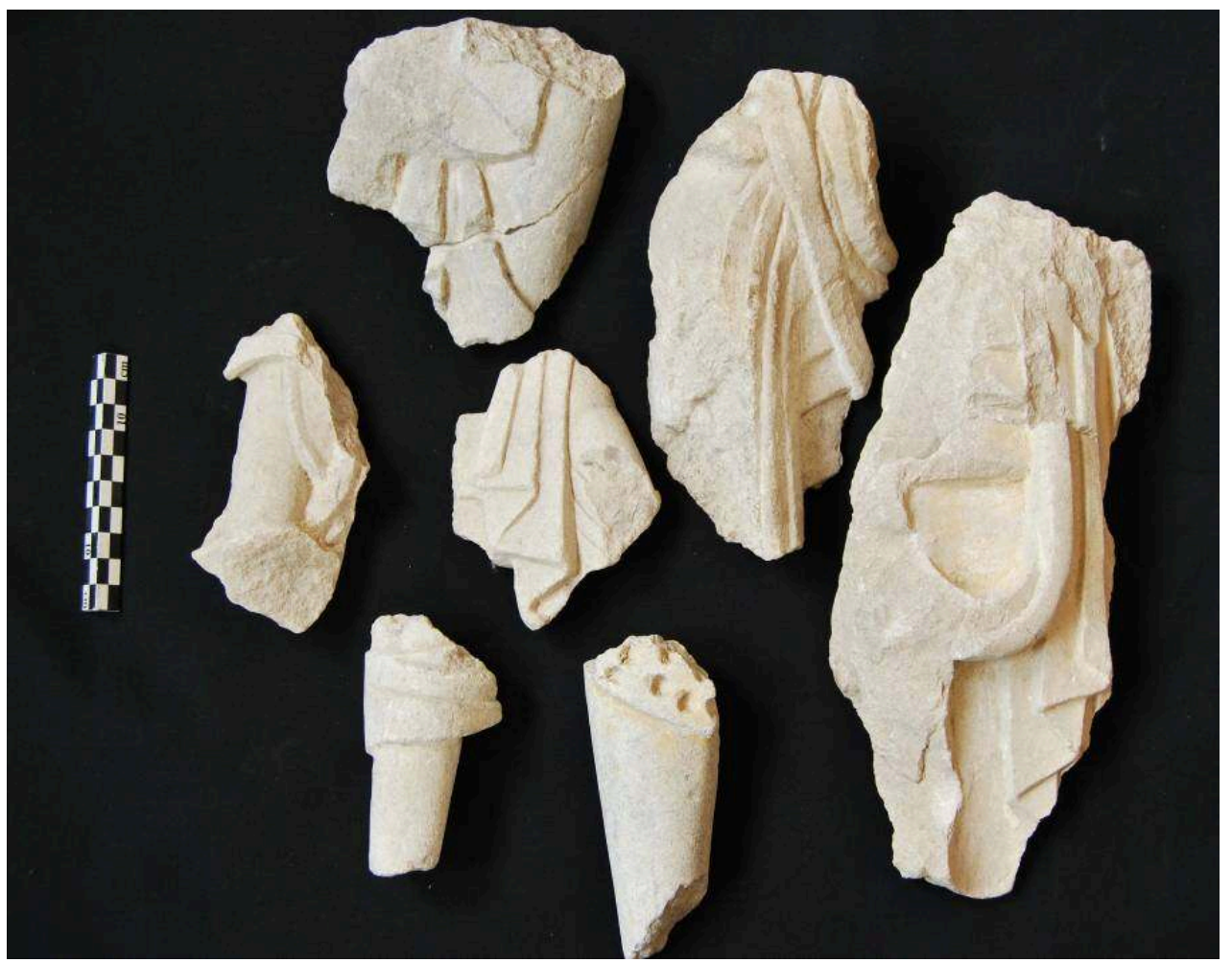

Fig. 6 - Cluny, portail roman, fragments du linteau : apôtres (cl. J. Rollier).

La plupart des personnages sont debout, excepté l'un d'entre eux, assis et tenant un livre. L'étude archéologique permet de reconnaître au moins quatre personnages supplémentaires, ce qui fait au total une quinzaine de figures pour le linteau, alors 
qu'au milieu du XVIII ${ }^{\mathrm{e}}$ siècle Benoît Dumolin en avait compté vingt-trois : «La face de la couverte est ornée de vingt-trois figures de saints en relief, presque entassées tant elles sont proches l'une de l'autre. » Malheureusement, il n'est plus possible de restituer la coloration de leurs vêtements, la couleur jaune qui subsiste appartenant à la préparation. Dans les meilleurs des cas, nous observons des restes infimes de couleurs, notamment des restes rouges sur un revers de manteau ${ }^{24}$.

Seule la tête du soldat endormi, appartenant peut-être à la scène des saintes femmes au tombeau, conserve une polychromie presque intacte. La carnation est rose sur préparation jaune, le bord inférieur des paupières est encore légèrement gris-noir (noir de carbone) et des restes de badigeon blanc indiquent qu'il a été recouvert par la suite. D'après H. Kleinschmidt, il subsistait des restes bleus sur la mentonnière, vestiges qui ont disparu depuis.

Un fragment particulièrement riche en informations est celui où figurent deux pieds d'apôtre posés sur le bord du linteau ${ }^{25}$. En effet, les pieds, jaunes, sont posés sur un sol vert et un fond bleu. Le motif végétal à feuille d'acanthe, qui court le linteau, est teinté de vert, avec de petits rehauts jaune vif, l'ensemble des feuillages se détachant sur un fond rouge.

L'observation sous loupe binoculairedes échantillons bleus met en évidence l'absence de pigment bleu ${ }^{26}$. Les matériaux détectés sont un mélange de blanc de plomb et de noir de carbone, ce qui est un substitut pour le pigment bleu, tel que nous le connaissons ailleurs en Bourgogne ${ }^{27}$. L'état de conservation est cependant trop abîmé pour émettre un avis définitif à propos de l'absence de bleu, d'autant plus qu'il est présent sur les voussures. Le gris-bleu n'est peut-être qu'une sous-couche permettant d'économiser le pigment bleu.

Le vert est un pigment au cuivre posé sur un fond rouge, à base de cinabre, sur un mélange de minium et de blanc de plomb. Les rehauts jaune vif sont obtenus à partir de stannate de plomb, ce qui indique un repeint post-roman.

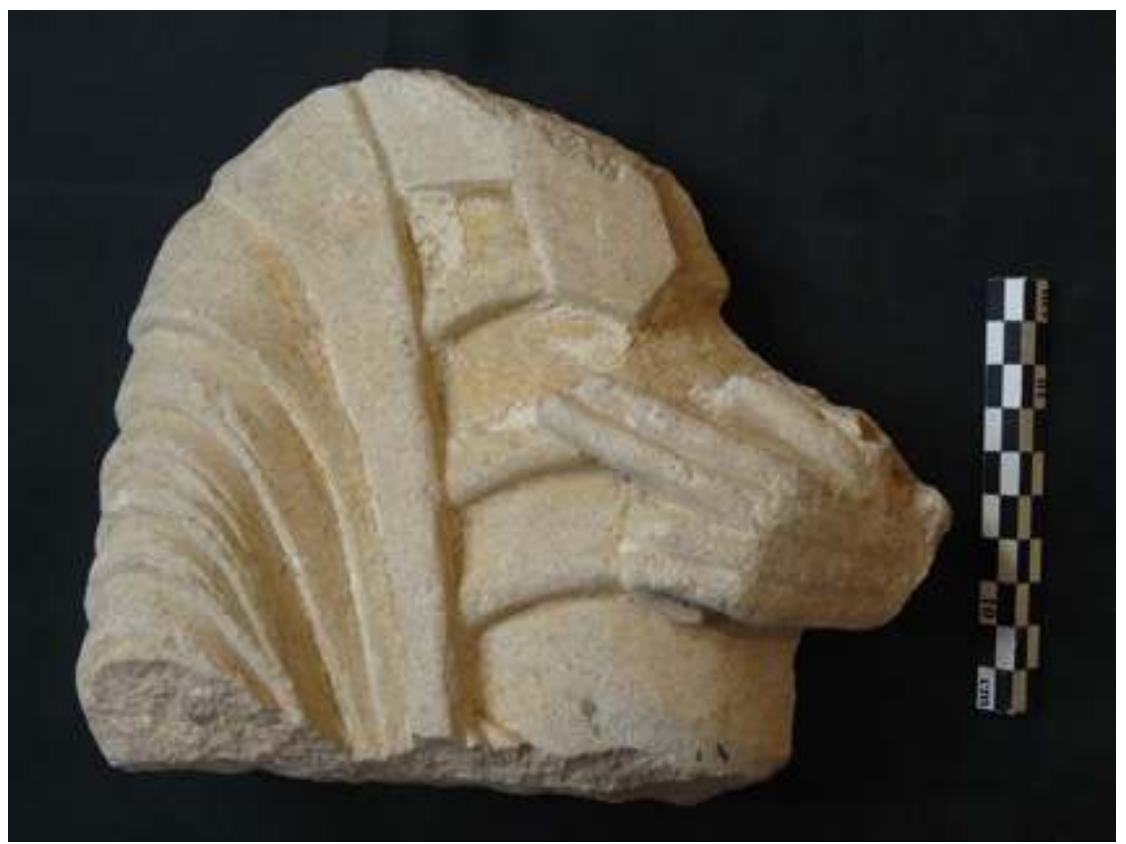

Fig. 7 - Cluny, portail roman, détail d’un apôtre : préparation jaune recouverte de badigeon blanc (cl. J. Rollier) 


\section{Les voussures (fig. 8)}

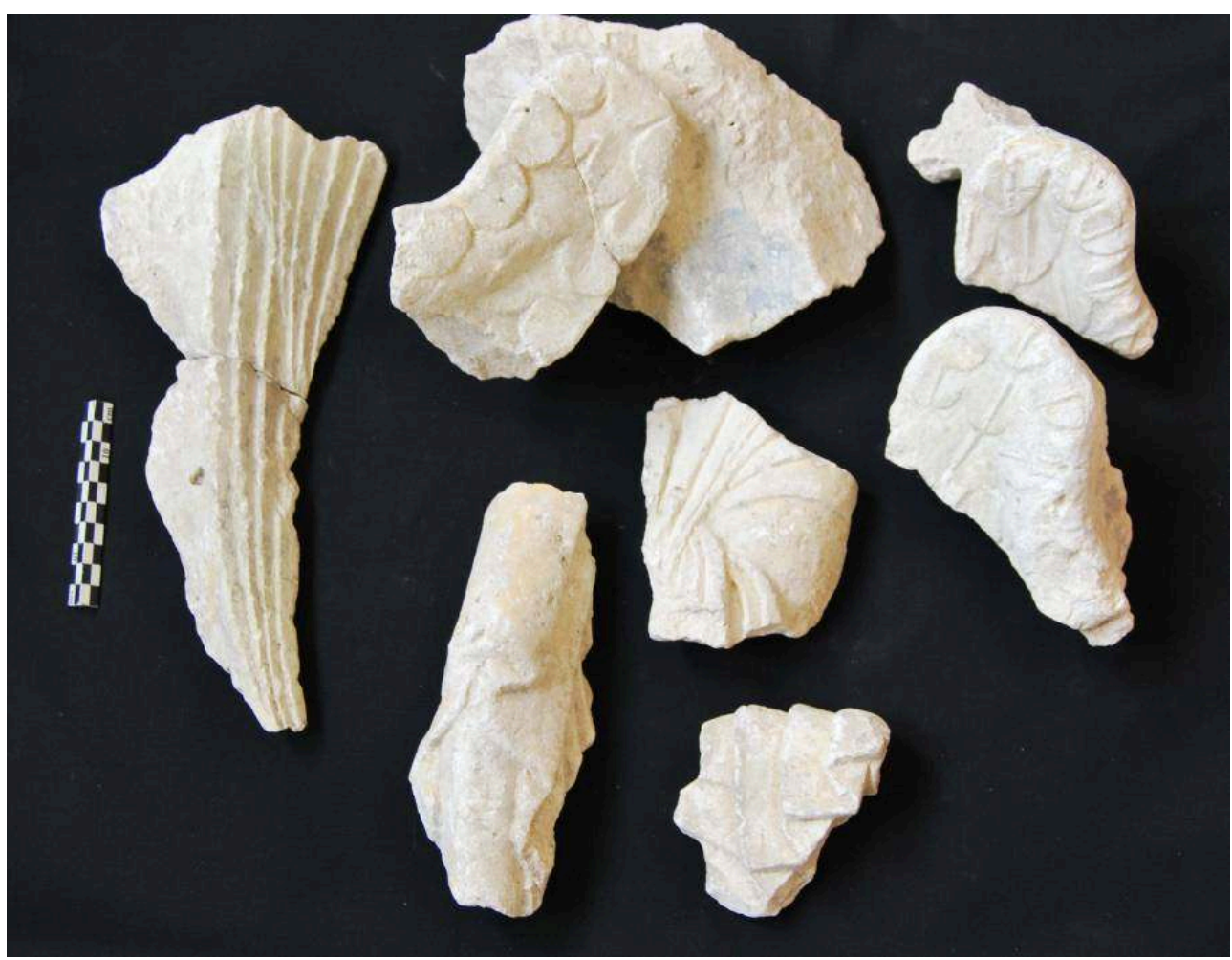

Fig. 8 - Cluny, portail roman, voussure des anges, éléments d'ailes et de drapés (cl. J. Rollier).

L'archivolte comptait cinq voussures qui sont décrites avec une certaine précision par Benoît Dumolin, ce qui nous a permis de retrouver les différents éléments parmi les centaines de fragments triés. Le calcaire blanc de la Lie, utilisé pour trois des voussures, est plus tendre et plus facile à sculpter que celui utilisé pour les autres parties du portail, ce qui explique en partie une meilleure adhérence des couleurs. Nous avons ainsi rassemblé deux cent vingt fragments appartenant à l'ensemble des voussures, auxquels il faut ajouter les éléments inclus dans des reconstitutions en plâtre de Conant. En 1970, Kleinschmidt observait des dorures sur la première voussure (motif à fleurs) et la seconde voussure (nimbes des anges), ce que nous avons cherché à vérifier. Elle observait également des cheveux roussâtres et du rouge sur les têtes d'anges, ainsi que des ailes vertes.

La première voussure était composée de fleurs à cinq pétales, dont certains sont bien conservés et attestent d'une succession de trois couches colorées et d'un badigeon blanc. La première strate, probablement romane, était dorée, tandis qu'un repeint est bien perceptible par sa matière craquelée (liant gras?) et ses couleurs très vives, à dominantes rouge vermillon, qui recouvrent complètement les dorures. Plusieurs pétales conservent ainsi une stratigraphie complexe avec la préparation au blanc de plomb, une couche d'ocre jaune et la dorure romane ${ }^{28}$.

La deuxième voussure était occupée par quinze anges sous des arcatures. Plusieurs fragments de visages sont préservés, ainsi que des éléments de nimbes qui conservent des restes de feuille d'or, posée sur une préparation rouge vif ${ }^{29}$. Un visage conserve encore un œil noir et des cheveux colorés en rouge-brun. 
31 Des éléments de carnations (mains, pieds) indiquent une coloration jaune uniforme, très pulvérulente. Un fragment plus important est constitué d'un pied jaune posé sur une large bordure bleue et rouge. Ici, une sous-couche grise - mélange de blanc de plomb et de noir de carbone - permet d'économiser le précieux lapis-lazuli. Plusieurs fragments d'ailes et de vêtements sont peints en jaune ou en vert et se détachent sur un fond bleu vif, partiellement caché sous les badigeons blancs des réfections postérieures. La troisième voussure comportait un motif végétal, dont nous possédons encore plusieurs fragments, mais aucun prélèvement de couleur n'a été effectué sur la préparation jaune.

a quatrième voussure présente une série de vingt-cinq médaillons contenant des têtes de vieillards, dont trois sont conservées au musée. Les visages sont encore partiellement colorés en jaune, avec des détails rouges (sourcil, cheveux) et noirs yeux, cheveux, barbe et moustache. La technique de réalisation des yeux varie d'un morceau à l'autre, les uns étant en relief, les autres creusés, avec dans les deux cas une pupille peinte en noir.

34 La disposition des têtes dans des médaillons, telle que l'a proposée Conant, peut prêter à discussion. Les fragments de besants semblent avoir été placés de façon arbitraire dans le plâtre, morceaux qui pourraient également appartenir à certains chapiteaux de l'avant-nef. La description de Benoît Dumolin n'apporte pas de détail à ce sujet.

La cinquième voussure était lisse et les fragments n'ont pas fait l'objet de prélèvements.

Il résulte de toutes nos observations que le portail a été coloré une première fois à l'époque romane, puis repeint, vraisemblablement, à l'époque gothique, avec des couleurs posées à l'aide d'un liant gras ${ }^{30}$. Il ne s'agit pas de quelques corrections dans la polychromie d'origine, mais d'une reprise complète des décors.

Rappelons que le portail roman a été mis en place vers 1115-1120, suivi d'une longue construction des cinq travées de l'avant-nef. Au moment de la réalisation de la façade gothique, vers le milieu du XIII ${ }^{e}$ siècle, une reprise du portail roman était probablement nécessaire pour rafraîchir la façade empoussiérée par un long chantier.

Il en fut ainsi à la cathédrale de Bourges, où un premier portail, monté vers 1225 contre la façade sud, a été polychromé et utilisé tout au long de l'avancement du chantier, puis repeint quelque trente ans plus tard lorsque tous les autres portails de la façade ouest furent terminés ${ }^{31}$.

39 Après les périodes fastes du Moyen Âge, le portail roman de Cluny a été entièrement badigeonné en jaune, peut-être au XVII ${ }^{e}$ siècle, après les guerres de Religion, puis complètement recouvert de deux couches blanches, probablement au xvIII siècle, période de grands travaux à Cluny. Lorsque le portail a été recouvert, les polychromies étaient déjà très lacunaires, comme l'indique la matière terreuse qui se trouve entre les couleurs anciennes et les réfections.

\section{Les jambages}

De grands éléments appartenant aux jambages latéraux nous sont parvenus, dont certains sont encore partiellement colorés (fig. 9). Une bonne soixantaine de fragments peuvent être attribués à ces parties du portail, morceaux issus des fouilles de Conant ou des découvertes plus récentes ${ }^{32}$. 


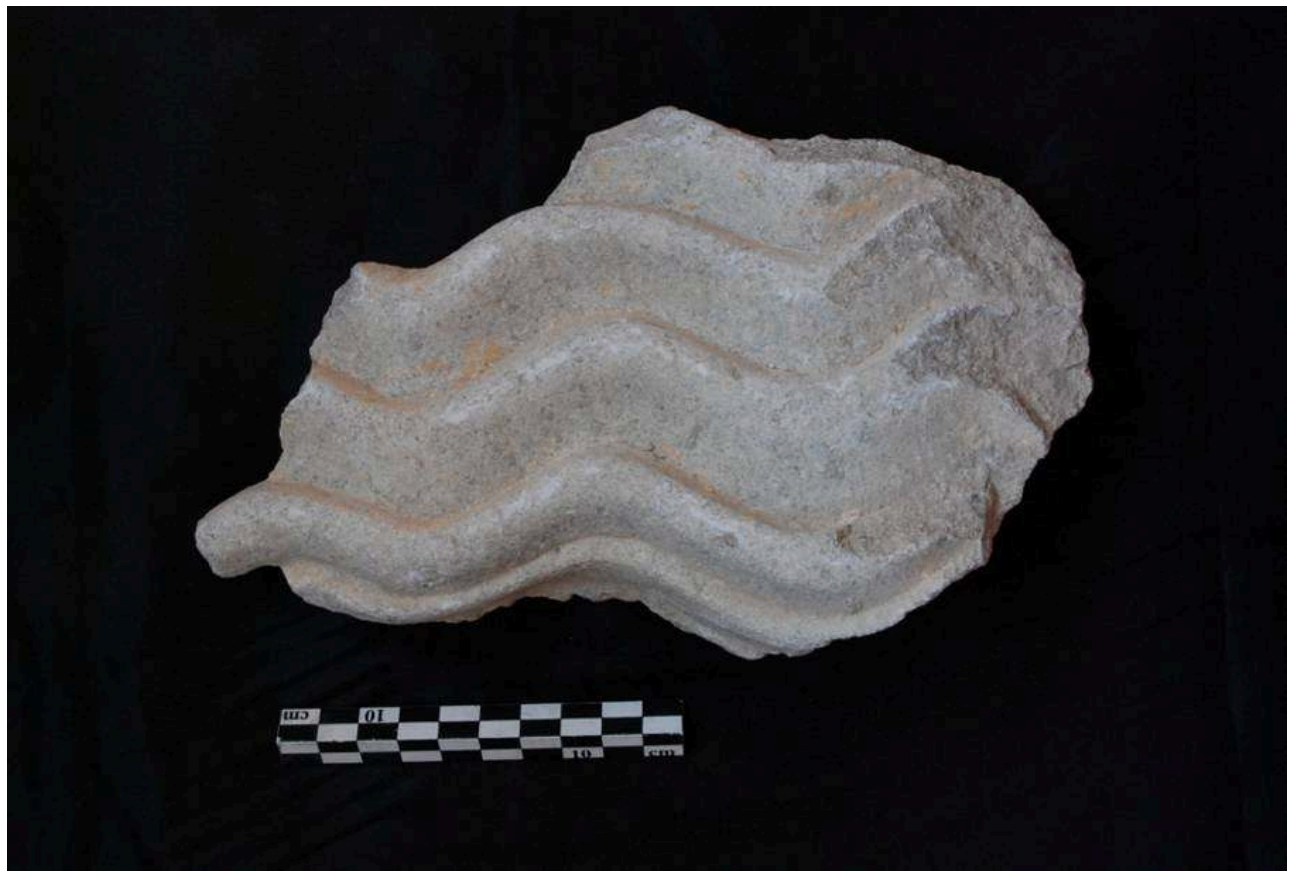

Fig. 9 - En haut : fragment des nuées des anges (inv. III-19888-III.962).

En bas : détail d'un élément de jambage (inv. 78.42-78.43), entre les deux corolles d'un médaillon (droite). Images en lumière naturelle.

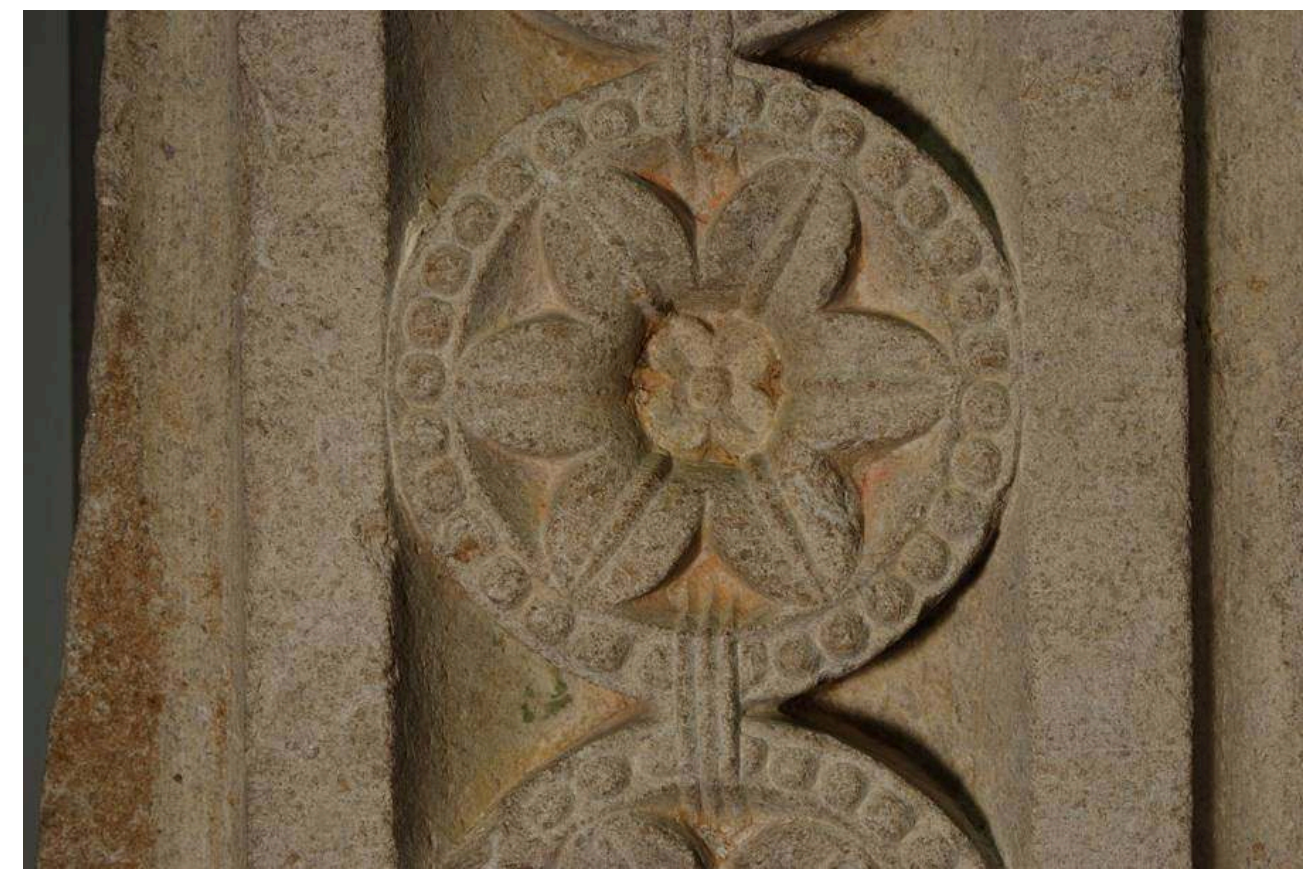

La stratigraphie est similaire : la feuille d'étain est posée sur une couche colorée (cl. S. Castandet). 

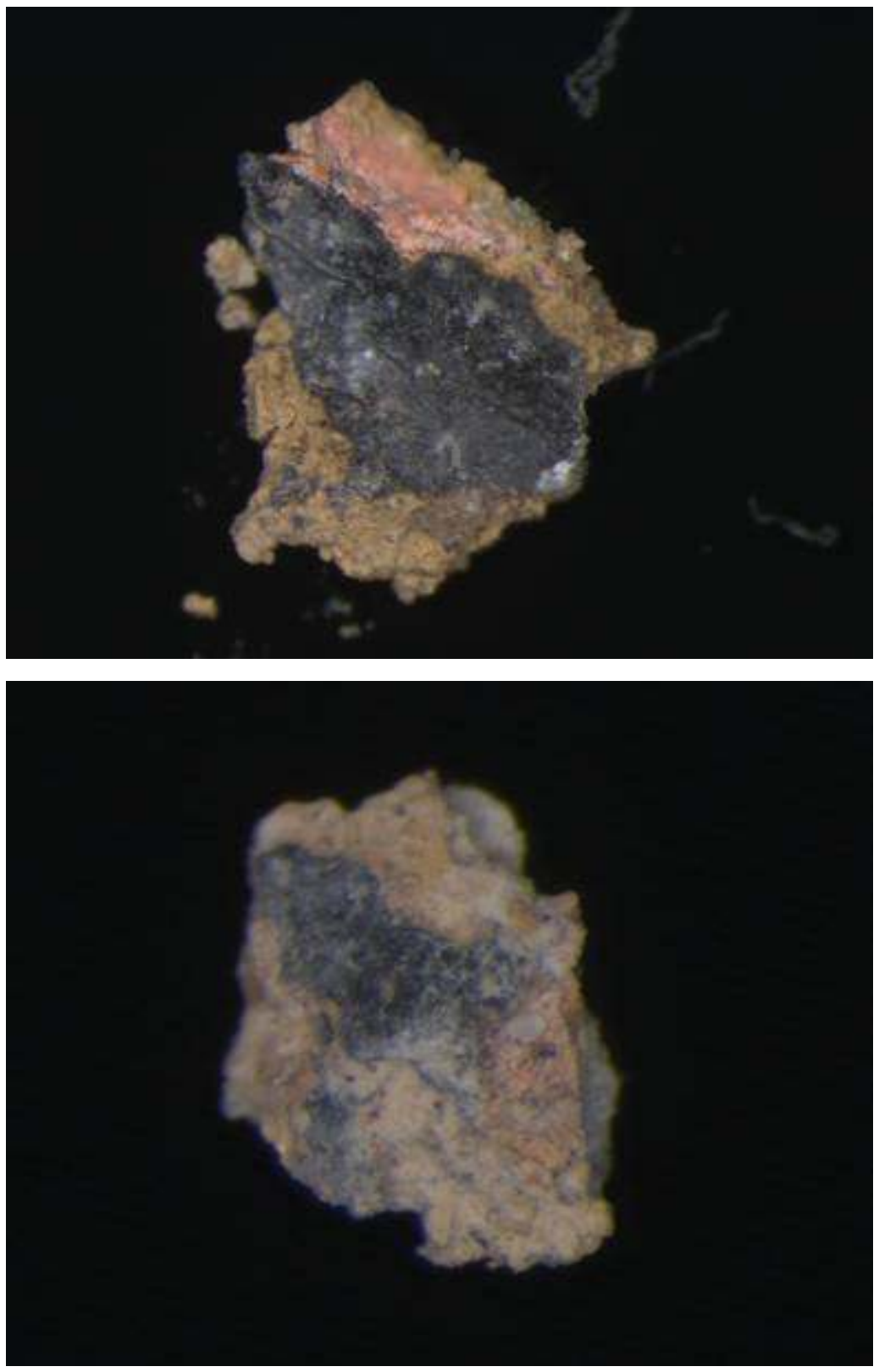

41 La description de B. Dumolin indique clairement l'ordre dans lequel il faut restituer les colonnettes et les piédroits. Nous savons ainsi que «l'arête des jambages est ornée du haut en bas d'un feuillage en manière de feuille d'eau [...] que quatre colonnettes se trouvaient de part et d'autre du portail [...] les trois premières de chaque côté sont d'autant plus remarquable qu'elles sont d'un seul morceau; les deux plus voisines de la porte sont taillées en réseau; les secondes en vis; les troisièmes chargées de roses placées dans les cannelures ; les dernières sont unies [...] ».

Les montants de la porte étaient entourés d'un décor à feuille d'acanthe, dont il reste un gros bloc présenté au musée, quatre gros blocs et plus de cinquante fragments provenant de notre tri. Selon Kleinschmidt, les feuillages verts se détachaient sur fond rouge, tout comme les grappes vertes, ce qui est encore vérifiable actuellement. Le petit motif de losanges était jaune. Le détail des polychromies a été donné dans le paragraphe sur le linteau. 

plus loin dans la reconstitution de cet ensemble détruit. L'aspect des maçonneries environnantes a été rendu par texturation informatique de haute qualité, en se basant sur les éléments d'enduits médiévaux conservés dans le bras sud du grand transept. La société On-Situ a cherché à créer une ambiance intérieure aux teintes veloutées, en harmonie avec les couleurs vives du portail roman ${ }^{33}$.

\section{Conclusion}

Malgré l'état fragmentaire du portail roman de Cluny III, il est encore possible d'étudier les différentes parties de cette œuvre détruite avec brutalité. L'analyse des polychromies s'avère riche en information, avec une couche romane aux couleurs très vives - ocre jaune, rouge vermillon, minium, vert, bleu de lapis-lazuli -, rehaussées de dorures (or et étain) à la fois sur le tympan - mandorle du Christ, nimbes, nuées -, les voussures - motif floral, nimbes des anges -, les jambages. Le portail a ensuite été 
entièrement repeint, probablement à l'époque gothique, au moment de l'achèvement de la façade au milieu du xiIl ${ }^{e}$ siècle. Les fragments de cette façade gothique, polychromée à deux reprises, sont actuellement en cours d'étude ${ }^{34}$.

Le chantier de l'avant-nef de Cluny III s'est échelonné depuis 1115 jusqu'au XIII ${ }^{\mathrm{e}}$ siècle, nécessitant probablement des reprises. Deux travées sont encore romanes, tandis que les trois suivantes sont gothiques, ce qui signifie un changement de parti dans les élévations. La polychromie permettait d'harmoniser l'architecture, tout en rythmant les différents niveaux de l'élévation. Des restes colorés subsistent de façon très ténue sur la grande frise qui courait au-dessus du portail et le long des parois hautes de la nef. Dans l'état actuel de nos recherches, nous avons retrouvé quelques fragments de pétales de fleurs jaune et rouge, qui ressemblent à la grande frise de la cathédrale Saint-Lazare d'Autun (paroi haute de la nef centrale), où les fleurs sont placées sur une succession de carrés jaunes et rouge, dont la date est difficile à déterminer (fig. 10).

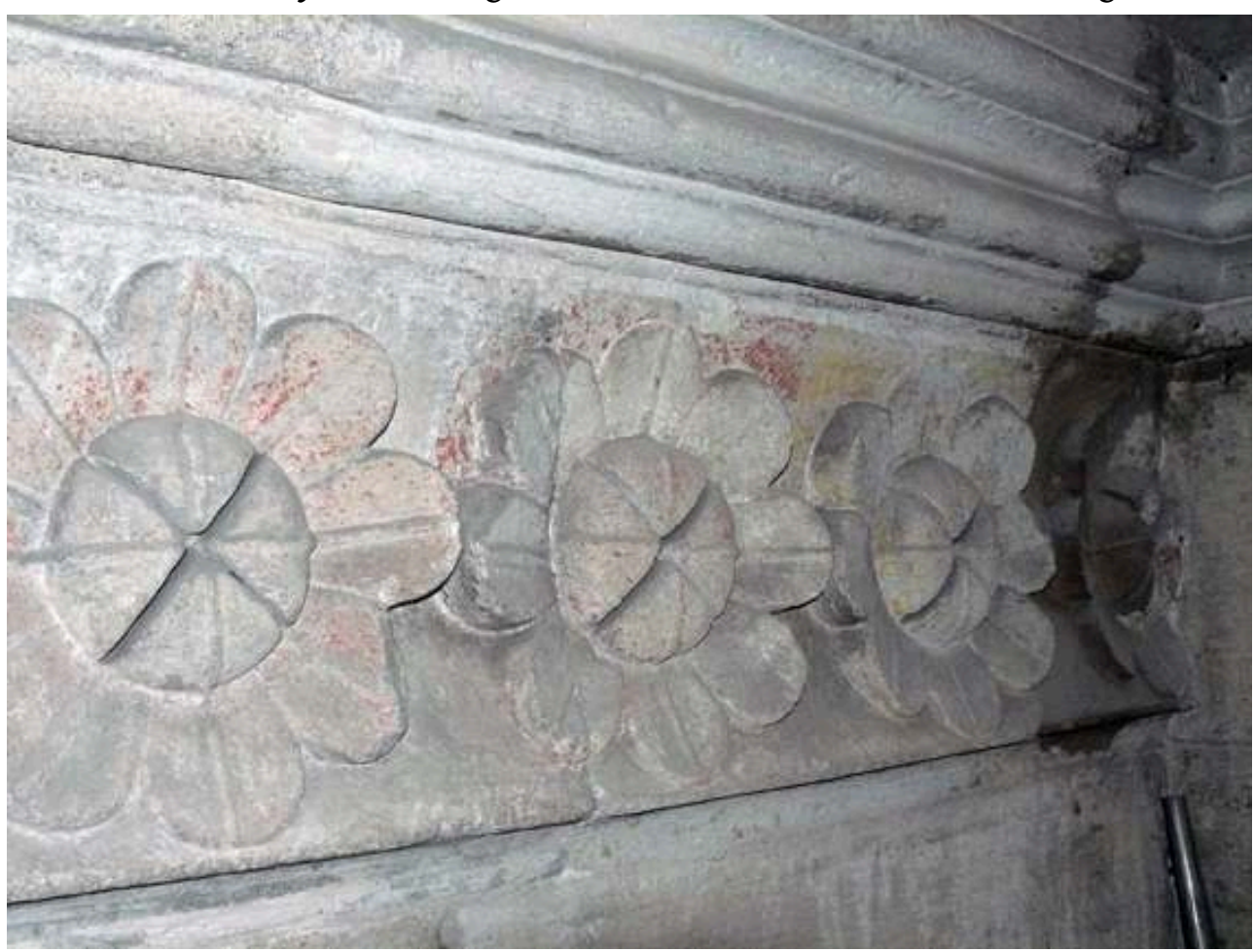

Fig. 10 - Cathédrale d'Autun, grande frise de la nef centrale.

Le matériel trouvé dans l'avant-nef de Cluny contient aussi quelques fragments de pilastres cannelés, qui alternent le rouge et le noir, tandis que de nombreux éléments sont simplement badigeonnés en blanc. Il semblerait que de grandes surfaces aient été blanches ${ }^{35}$ et que seuls les éléments architectoniques aient été peints.

\section{NOTES}

1. É. VERGNOLLE, L'art roman en France, Paris, 2003 (1 ${ }^{\text {re }}$ éd. 1994), p. 238. 
2. B. Dumolin, Description historique et topographique de la ville, abbaye et banlieue de Cluny, Cluny, 1749-1798 (musée d'Art et d'Archéologie de Cluny, ms. 71).

3. G. ROLLIER, Rapport de synthèse-fouilles de sauvetage, 1988-1990, Narthex de Cluny III et cour abbatiale (non publié); G. RolLIER, «Les fouilles archéologiques de l'avant-nef », Cahiers du musée d'Art et d'Archéologie de Cluny, juillet 1996, p. 16-21.

4. F. SALET, Cluny et Vézelay, l'œuvre des sculpteurs, Nogent-le-Rotrou, 1995.

5. D. Vingtain, L'abbaye de Cluny, centre de l'Occident médiéval, Paris, 1998, p. 88 (PARIS, BnF, Cabinet des estampes, coll. Destailleur).

6. Collection Vanuxem, reproduit dans K. J. ConAnT, Cluny, les églises et la maison du chef d'ordre, Mâcon, 1968, fig. 27.

7. PARIS, BnF, Cabinet des estampes, coll. Destailleur, publié dans A. BAUD, Cluny, un grand chantier médiéval au chœur de l'Europe, Paris, 2003, p. 89.

8. K. J. conant, Cluny..., op. cit.

9. Fouilles CONANT, fragment $n^{\circ} 1033$.

10. H. KLeInSChmidit, "Notes on the Polychromy of the Great Portal at Cluny ", Speculum, 45 (1970), p. 36-39.

11. J. Rollier-Hanselmann,membre de l'équipe Gunzo, chargée des vérifications archéologiques et de la restitution du portail pour la nouvelle maquette 3D de l'abbaye, version 2010 ; J. RoLLIERHANSELMANN, «Reconstitution des portails de Cluny III : des fouilles de Conant à l'imagerie virtuelle en 3D», Bulletin du Centre d'études médiévales d'Auxerre, 13 (2009), p. 157-170 [http:// cem.revues.org/index11058.html].

12. S. Castandet, doctorante, Arts et Métiers ParisTech CER de Cluny, IRAMAT-CRP2A (Institut de recherche sur les archéomatériaux et Centre de recherche en physique appliquée à l'archéologie), UMR 5060 (CNRS et université de Bordeaux 3).

13. Fouilles ROLLIER, 1989 , voir note 4 , fragment $n^{\circ}$ III-333.

14. Fouilles CONANT, fragment $n^{\circ} 242.7$.

15. Théophile donne une recette de vernis coloré afin de «dorer » l'étain, cf. M. THÉOPHILE, Essai sur divers arts, publié par le Cte Charles de l'Escalopier, Laget, Nogent Le Roi (XII'), rééd. de 1996, chap. XXVI.

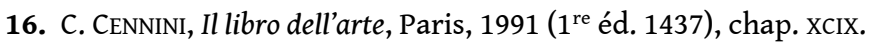

17. I. PALLOT-FROSSARD, «Polychromies des portails sculptés médiévaux en France. Contributions et limites des analyses scientifiques", in La couleur et la pierre. Polychromie des portails gothiques,Paris, 2002, p. 73-84, part. p. 81.

18. Le musée d'Art et d'Archéologie conserve une copie en plâtre (H. $63 \mathrm{~cm})$.

19. H. H. KLEINSCHMIDIT, «Notes... », op. cit., 1970.

20. Fouilles CONANT, fragment $n^{\circ} 1028(14 \times 10 \mathrm{~cm})$.

21. Fouilles CONANT, fragments $n^{\circ} 218,237.6$. L'avant-cuisse de l'animal n'a pas été retrouvée.

22. F. PEREGO, Dictionnaire des matériaux du peintre, Paris, 2005.

23. Nous comptons 57 fragments, auxquels nous pourrions en rajouter d'autres, plus difficiles à classer.

24. Fouilles CONANT, fragment $n^{\circ} 243.3$. Nous observons la stratigraphie suivante: préparation jaune, puis couche rouge sur le revers du manteau, recouverte d'une matière terreuse, puis de badigeon blanc.

25. Il s'agit de trois fragments recollés par K. J. CONANT, fragments $\mathrm{n}^{\circ \mathrm{os}} 214,228.63,232.7$.

26. Le pigment bleu a été recherché par différentes méthodes, sous microscope optique, puis sous microscope métallographique et sous spectre Raman. Le MEB a détecté très peu de cuivre, de l'ordre de $2 \%$.

27. J. ROLLIER-HANSELMANN, «D'Auxerre à Cluny: techniques de la peinture murale entre le viII et le XII ${ }^{\mathrm{e}}$ siècle en Bourgogne», Cahiers de civilisation médiévale, 40 (1987), p. 57-90. 
28. Fouilles CONANT, fragments $\mathrm{n}^{\text {os }} 1052.51,1044.69,1034.50$.

29. Fouilles CONANT, fragments $\mathrm{n}^{\mathrm{os}} 1035.59,1035.29,1044.74$.

30. Fouilles CONANT, fragments $n^{\circ} 232.16$ et 212.5 (fragment de jambage, avec repeint); stratigraphie: préparation jaune recouverte d'une couleur rouge vif (décor roman), puis d'un vert rehaussé de jaune citron (décor gothique). Un badigeon blanc a ensuite recouvert toutes les couleurs antérieures.

31. R. ROSSI MANARESI et A. TUCCI, "The polychromy of the portals of the gothic cathedral of Bourges ", Preprints ICOM Committee for Conservation, Copenhague, 1984, 84,5. 1-4; R. ROSSI MANARESI, "Observations à propos de la polychromie de la sculpture monumentale romane et gothique», in La couleur et la pierre. Polychromie des portails gothiques, Paris, 2002, p. 57-64.

32. Parmi ces éléments, il y a 5 gros blocs présentés dans la salle inférieure du musée, 6 petits fragments dans une petite vitrine temporaire, auxquels nous ajoutons une cinquantaine d'éléments retrouvés dans les réserves. Un gros bloc vient d'être découvert en hiver 2009, lors des travaux de pavement de la place du 11 août 1944 à Cluny.

33. Jean-Michel Sanchez est également chargé des installations muséographiques de l'abbatiale de Romainmôtier (Suisse), du Louvre-Lens pour ne citer que ces cas. De nombreuses autres réalisations muséographiques ont été réalisées par l'équipe d'On-Situ à Chalon-sur-Saône, notamment la reconstitution polychrome du Beau-Dieu d'Amiens (musée des Monuments français) ou la mise en espace interactive du scriptorium du Mont Saint-Michel, musée d'Avranches.

34. Gaëlle Ferdek, étudiante en Master 1 à l'université de Bourgogne, a entrepris cette étude sous la direction du professeur Daniel Russo (titre du mémoire: Étude, conservation, restauration et restitution du portail gothique de Cluny III).

35. Lors des fouilles de l'avant-nef, de grandes quantités d'enduit peint en blanc ont été retrouvées.

\section{INDEX}

Index géographique : France/Cluny

Mots-clés : abbaye, portail, restitution, couleur 